\title{
Using Fourier spectroscopy for the assessment of oxidative degradation of polypropylene medical meshes
}

\author{
Nikolay P. Kozlov, and Rafael I. Fairushin* \\ Optics and spectroscopy Department, Samara National Research University, 34 Moskovskoe shosse., Samara 443086, \\ Russia \\ * e-mail: rafael.fairushin2014@yandex.ru
}

\begin{abstract}
We considered the effect of oxidative degradation of polypropylene (PP) on its spectral properties. For this aim, the model experiments have been performed on the oxidation of PP mesh by the exposure to UV radiation and the technique of quantitative assessment of the appeared oxygen-containing groups has been elaborated. As a result, the concentrations of carbonyl and hydroxyl groups were found and the detection ability of the technique was estimated. (C) 2017 Journal of Biomedical Photonics \& Engineering.
\end{abstract}

Keywords: IR Fourier spectroscopy; polypropylene mesh; oxidation of polypropylene.

Paper \#3140 received 24 Dec 2016; revised manuscript received 10 May 2017; accepted for publication 10 May 2017; published online 1 Jun 2017. doi: 10.18287/JBPE17.03.020310. [Special Issue. Workshop "Biophotonics" of the XIII all-Russian Youth Samara conference-contest on optics and laser physics].

\section{References}

1. O. V. Vostrikov, Experimental study of interaction between the tissues and mesh implants used in abdominal hernia surgery, Diss. Cand. Sci. in Med., Novosibirsk, Russia (2006) [in Russian].

2. D. V. Kuznetsov, D. S. Muratov, V. V. Cherdyntsev, I. A. Ilyinykh, N. V. Shatrova, and I. N. Burmistrov, "Study of resistance of polymer composites based on polypropylene to ultraviolet radiation," Sovremennye problemy nauki i obrazovaniya 6 (2012) [in Russian].

3. J. L. White, and D. D. Choi, "Polyolefins: Processing, Structure Development, And Properties," Hanser Verlag, Munich (2005). ISBN 1569903697

4. L. Balaban, I. Mayer, "Photooxidation destruction of polypropylene," Materials of International Symposium on Polymers, Mir, Moscow, 307-309 (1967) [in Russian].

5. J. Dechant, R. Danz, W. Kimmer, R. Schmolke, "Ultrarotspectroscopische Untersuchungen an Polymeren," Akademie Verlag, Berlin (1972) [in German].

6. A. V. Klimenko, V. M. Zorin, "Heat Power and Heat Engineering. General Problems: A Handbook," MEI, Moscow (1999) [in Russian].

\section{Introduction}

Due to high chemical inertness and good biocompatibility, PP is extensively used in many fields of vital activity, including medicine. However, in medical practice it sometimes happens so that the organism rejects the mesh, which leads to unwanted sequela. A hypothesis exists that this is due to PP degradation in the process of its oxidation under the effect of the living organism environment [1].

A specific feature of the study is that the object of study is a mesh used in medical practice rather than a film, as in Ref. [2]. The samples extracted from the organism and accordingly the control samples cannot be converted into powders or films in order to reveal the structural changes, caused by the effect of living organism, without introducing possible changes related to the additional mechanical impact. In contrast to films, meshes have holes, so that only a fraction of the IR radiation incident on the sample passes through PP. This feature requires special technique to process the spectra of such meshes.

The aim of the present paper is to analyse the oxidative degradation of PP using IR spectroscopy. 


\section{Theoretical background and experimental technique}

$\mathrm{PP}$ is a high-molecular polymer synthesised by stereospecific polymerisation of propylene under rather low pressure using the Natta-Ziegler catalysts. Different conditions of the polymerisation process yield the production of polymers with different molecular structure that determines their physical and spectral properties. Depending on these conditions, a few types of PP structures can be obtained that differ in the spatial localisation of methyl groups with respect to the main polymer chain: the isotactic, syndiotactic, and atactic structures [3]. For mesh production, the isotactic structure is used, since it possesses better mechanical and chemical properties.

The main instrument for experimental studies is the Fourier spectrometer FSM 2201. In the experiment, we used the PP mesh with the fibre thickness $0.1 \mathrm{~mm}$. During the spectrum recording, the samples were fixed with holders placed in the cuvette section of the spectrometer. The spectra were measured in the transmission mode with the resolution $1 \mathrm{~cm}^{-1}$. The studied spectral region was from 400 to $4000 \mathrm{~cm}^{-1}$. Each final spectrum was obtained by averaging over 20 individual scans. Before studying each sample, the reference spectrum was recorded.

To model the oxidative degradation of PP mesh, the sample was exposed to UV radiation. The irradiation of the sample was performed using the arc mercury lamp DRT 230. The light flux power density calculated using the spectral characteristics of the mercury lamp amounted to $0.3 \mathrm{~W} / \mathrm{cm}^{2}$. The exposure was executed in a few stages, after each stage the transmission spectrum of the sample was recorded. The duration of each stage was one hour. The total exposure time was 10 hours.

\section{Experimental results}

In the course of experiment, the PP mesh extracted from a human organism was studied. Figure 1 makes it possible to see that in the spectrum of the extracted sample at the wavenumbers 668, 2343 and $2360 \mathrm{~cm}^{-1}$, corresponding to water, the decrease of absorption band intensities was observed. This is due to different absorption capability of the samples' surface. One can also observe the difference in the absorption saturation, which is explained by the normalisation of the spectra to the maximal transmission. Such normalisation is necessary for comparison, since the transmission of samples is essentially different. In the rest of the spectrum no characteristic changes, typical for functionally substituted PP were detected.

Since in the studied samples no results of oxidative degradation were found, it was decided to compare the results with the known results of the PP oxidative degradation under the exposure to UV radiation [4]. In this case, there are the oxidation results in the appearance of hydroxyl and carbonyl groups in the polymer chain.

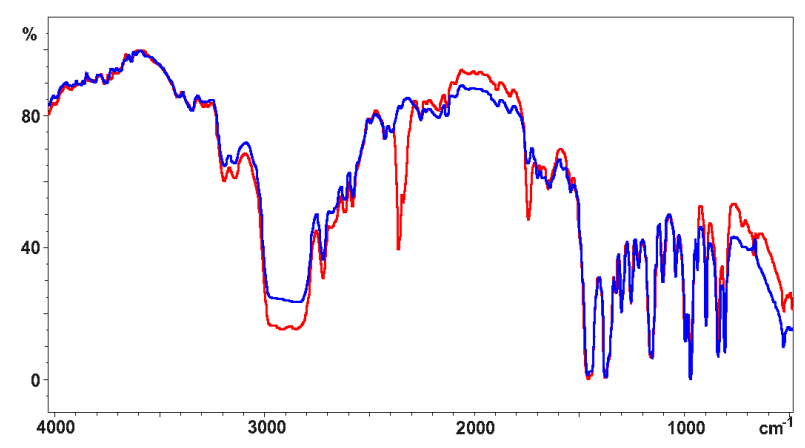

Fig. 1 Spectra of the extracted PP mesh (blue) and the initial PP mesh (red).

The analysis of the spectra has shown that in the spectrum of PP mesh exposed to UV irradiation during 10 hours a number of bands are observed that evidence in favour of the PP degradation process. However, in correspondence with the aim of the present work, we are interested only in those bands, which are related to the oxidative destruction of the polymer. The presence of these bands in the spectrum is caused by the appearance of hydroxyl and carbonyl functional substitution groups.

In the region $3300-3500 \mathrm{~cm}^{-1}$ of the irradiated PP mesh transmission spectrum (Fig. 2), one can observe a dip, which, according to the published data, is related to the appearance of hydroxyl groups, characteristic for the oxidation process in this polymer. We recorded the spectrum of vaporous ethanol $\left(\mathrm{C}_{2} \mathrm{H}_{5} \mathrm{OH}\right)$, having the hydroxyl group, and compared it with the spectrum of the studied sample. The centres of the broad absorption band $\left(3400 \mathrm{~cm}^{-1}\right)$ in the spectra of the irradiated mesh and ethanol practically coincide.

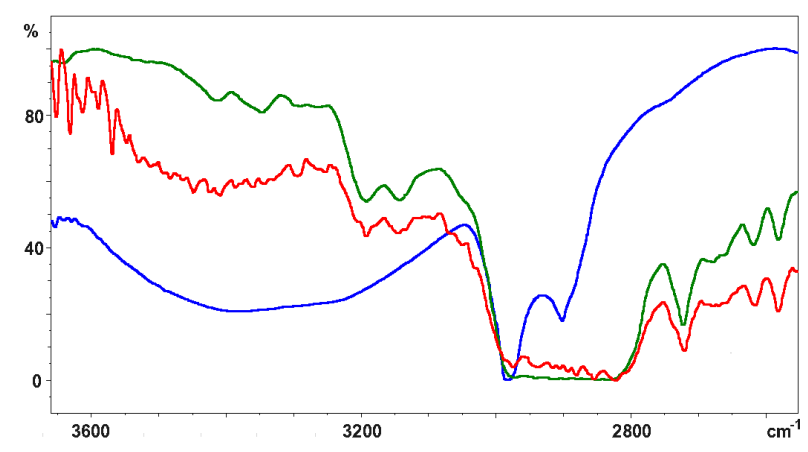

Fig. 2 Spectra of ethanol (blue), original PP mesh (green), and PP mesh exposed to UV radiation during 10 hours (red).

Besides that, in the absorption spectrum the absorption lines corresponding to the wave number $1715 \mathrm{~cm}^{-1}$ were found (Fig. 3). These lines were attributed to the vibrations of carbonyl groups. The origin of these lines is also related to the oxidative degradation. As a reference sample the stearin acid $\left(\mathrm{C}_{7} \mathrm{H}_{15} \mathrm{COOH}\right)$ was chosen, for which the presence of absorption lines in this region is characteristic. 


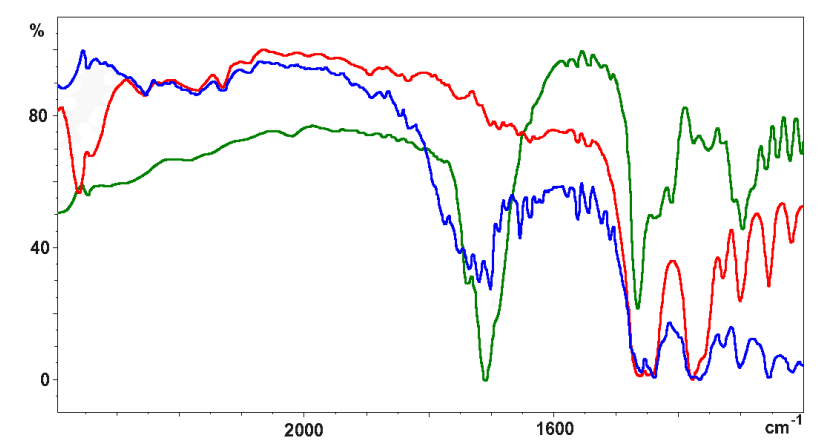

Fig. 3 Spectra of stearin (green), original PP mesh (red), PP mesh after 10 hours of exposure to UV radiation (blue).

\section{Quantitative concentration assessment of hydroxyl and carbonyl groups in the pp mesh after $U V$ irradiation}

For quantitative assessment of the carbonyl $(\mathrm{C}=\mathrm{O})$ and hydroxyl $(-\mathrm{OH})$ groups content in the mesh, exposed to the UV radiation, the data on the spectra of the characteristic substances known from Ref. [5] were used.

The absorption corresponding to certain vibrations can be related to the change in the optical density. The ratio of the change in the optical density of the studied sample $\Delta D$ to that of the reference sample $\Delta D_{0}$ is expressed as

$$
\frac{\Delta D}{\Delta D_{0}}=\frac{g n l}{n_{0} l_{0}},
$$

where $g$ is the mesh filling factor.

To determine this factor in the spectrum, we used the region of saturated absorption. In the spectrum, one can see that the value of transmission corresponding to this saturation amounts to $80 \%$. Correspondingly, the filling factor of the mesh equals $\sim 0.2$.

Besides that, an independent estimate of $g$ was found using the geometric characteristics of the mesh. We measured the volume of the mesh and its mass, which yielded the density equal to $0.16 \mathrm{~g} / \mathrm{cm}^{3}$. Since the density of bulk PP is known $\left(0.91 \mathrm{~g} / \mathrm{cm}^{3}\right)$, we get the same filling factor of the mesh $g=0.2$.

Form Eq. (1) the concentration $n$ is expressed as

$$
n=\frac{\Delta D n_{0} l}{\Delta D_{0} g l_{0}} .
$$

As a reference sample for the determination of concentration of carbonyl groups, we prepared a tablet of $\mathrm{KBr}$ containing $10 \%$ of stearin, which corresponded to the carbonyl groups concentration $n_{0}=5 \cdot 10^{20} \mathrm{~cm}^{-3}$. Measuring the appropriate optical densities in the reference sample and the studied one in the region of $1715 \mathrm{~cm}^{-1}$, we found $n=2 \cdot 10^{20} \mathrm{~cm}^{-3}$ in the studied sample.

For the hydroxyl group the saturated ethanol vapours were used as a reference sample, for which the relation between the pressure and the temperature is known [5]. Making use of the equation $n=P / k T$, we get the concentration of hydroxyl groups in the ethanol $n_{0}=1,227 \cdot 10^{18} \mathrm{~cm}^{-3}$. For calculation, the optical density at the $3400 \mathrm{~cm}^{-1}$ absorption line was used. The concentration of hydroxyl groups calculated from the appropriate spectra amounted to $n=2.6 \cdot 10^{18} \mathrm{~cm}^{-3}$ in the studied mesh.

In the obtained spectra, the signal levels in the region corresponding to carbonyl and hydroxyl groups for PP exceeded the noise level by nearly 5 times. This fact allowed the estimation of the minimal level of concentration of the groups that corresponds to the detection threshold. The obtained values are $n_{C=O}=10^{20} \mathrm{~cm}^{-3}, n_{-}{ }_{\mathrm{OH}}=1.5 \cdot 10^{18} \mathrm{~cm}^{-3}$, respectively. In other words, the technique allows the detection of one hydroxyl group per 15000 carbon atoms and one carbonyl group per 300 carbon atoms.

\section{Conclusion}

As a result of this work we arrived at the following results and conclusions:

- The technique of PP spectra recording was developed and the identification of spectral lines was carried out.

- The destruction of samples modelled by means of UV irradiation has shown that this process indeed is accompanied by oxidative reactions with the attachment of carbonyl and hydroxyl groups to the polymer chain. The concentrations of these groups were quantitatively estimated.

- The detection thresholds of the carbonyl and hydroxyl groups were assessed. It is shown that these values allow reliable detection of the appearance of the oxidation reaction products in PP, e.g., in the process of the polymer UV irradiation.

- For PP meshes degraded due to the action of living organism environment the concentration of products of oxidative reactions appeared to be smaller than the detection threshold, which made it impossible to judge unambiguously on whether the oxidative reactions are the dominant mechanism of PP degradation or not. 